Andy Buschmann

\title{
Protest und Demokratisierung in Myanmar: Erste Erkenntnisse aus dem „Myanmar Protest Event Dataset“
}

https://doi.org/10.1515/asia-2017-0064

\begin{abstract}
Myanmar has been perceived to be in a sociopolitical and economic transition since the semi-civilian government under President Thein Sein took office in 2011. Amongst other things, for a representative democracy to function, citizens have to have the freedom to express their opinions and deliberate them with fellow citizens. This requires the secure granting of fundamental civil liberties, as prescribed in the freedom of expression, association, and assembly. Hence, once in the process of democratization, a formerly authoritarian state has to make significant improvements in the granting and protection of these rights too. To empirically test whether Myanmar has made such improvements since 2011 is the goal of a greater research project I am working on. This research note introduces the project and summarizes first findings. By combining knowledge on legal reforms and protest data from the Myanmar Protest Event Dataset, it is suggested that, in Myanmar, the de jure exercisability and de facto exercise of fundamental civil liberties have significantly improved from 2011 to 2015. Informal methods of suppressing the right to protest, such as arbitrary violence, have increasingly descended while methods that are formally in accordance with the rule of law but still lack compliance with international human rights standards have ascended.
\end{abstract}

Keywords: Myanmar, protest, civil liberties, democratization, political transition, civil society

Nach Jahrzehnten der Isolation befindet sich Myanmar nun in einer Phase des sozialen, wirtschaftlichen und politischen Wandels. Mit der Wahl des Ex-Generals Thein Sein als Präsidenten im Februar 2011 wurden weitreichende Reformen auf den Weg gebracht. Diese Reformen haben unter anderem auch den Wahlsieg Aung San Suu Kyis und ihrer Partei, der National League for Democracy (NLD), im November 2015 ermöglicht. Viele Beobachter sehen Myanmar aus diesem Grund seit spätestens 2011 in einer politischen Transition, im besten Falle, in

Andy Buschmann, St Antony's College \& Department of Politics and International Relations, University of Oxford, Oxford, UK. E-mail: andy.buschmann@gmail.com 
Richtung Demokratie. ${ }^{1}$ Allerdings sind es nicht nur Wahlen und Parlamente die zu einer repräsentativen Demokratie, so wie sie sich viele (westliche) Regierungen in Myanmar wünschen, dazu gehören. Auch Freiheitsrechte und zivilgesellschaftliches Engagement sind substantielle Bestandteile von Demokratie. Es stellt sich also die Frage, wie sich diese Dimensionen in Myanmar seit 2011 verändert haben?

Wissenschaftliche Beiträge, die sich mit der Entwicklung von zivilgesellschaftlichem Engagement in Demokratisierungskontexten, speziell Protest, befassen existieren zwar, diese beschäftigen sich aber überwiegend mit den „neuen Demokratien“, welche nach dem Zusammenbruch der Sowjetunion in Ost- und Südosteuropa entstanden sind. ${ }^{2}$ Nur wenige Arbeiten haben konkrete Demokratisierungsbeispiele in Asien als Forschungsgegenstand, noch seltener betrachtet werden Protestereignisse in demokratischen Transitionen in Asien. ${ }^{3}$ Die Forschungsbeiträge zu Protesten in Myanmar waren bisher auf einzelne Fallbeispiele von sozialen Bewegungen beschränkt. ${ }^{4}$ Bis dato vollständig fehlte eine makroperspektivische Untersuchung von „Protest-Trends“, weshalb das Myanmar Protest Event Dataset einen wichtigen Beitrag zur Forschung leistet, dessen Relevanz über den Einzelfall Myanmar hinausgeht. Im vorliegenden Research Note werden erste Erkenntnisse aus diesem Datensatz beschrieben und Interpretationsansätze unternommen, die allerdings allesamt noch weiterer Analysen bedürfen. Damit verfolgt diese Research Note die Ziele, erstens, die Forschungsfrage und das Forschungsdesign des Gesamtprojekts vorzustellen und, zweitens, erste Erkenntnisse aus dem Datensatz zu präsentieren. Es folgt daher zunächst eine kurze theoretische Diskussion der Rolle von Freiheitsrechten in Demokratisierungskontexten, gefolgt von einer Analyse der Reformen von Freiheitsrechten in Myanmar sowie Einblicke in die Entwicklung von Protestereignissen.

\section{Die Bedeutung von Freiheitsrechten in Demokratisierung}

Folgt man gegenwärtigen Demokratietheorien, dann ist das Recht auf freie Meinungsäußerung, Assoziations- und Versammlungsfreiheit ein Muss, damit Bürger vom Staat unabhängige Meinungen bilden und diese öffentlich äußern

1 Egreteau 2012, 2016.

2 Siehe beispielsweise Ekiert/Kubik 2001 Oberschall 2000.

3 Eine Ausnahme sind die jüngeren Arbeiten zu Protest in Südkoreas Demokratisierung, siehe Chang 2015 Chang/Vitale 2013 Kim 2009.

4 Beispielsweise Chan 2017. 
können. ${ }^{5}$ Somit sind diese Freiheitsrechte eine notwendige Bedingung für bedeutungsvolle Wahlen. ${ }^{6}$ Freiheitsrechte erlauben darüber hinaus, dass eine aktive Zivilgesellschaft entstehen kann, welche kollektive Forderungen gegenüber dem Staat gebündelt artikuliert. ${ }^{7}$ Auch in „von oben“ gelenkten Regimewandeln (wie man ihn auch in Myanmar annehmen kann, da das Militär (Tatmadaw) die Reformen in 2011 eigenständig initiiert hat und weiterhin in vielen Bereichen Vetomacht besitzt ${ }^{8}$ ), können zivilgesellschaftliche Akteure den Druck für weitere Reformen in Richtung Demokratie erhöhen und deshalb ein wesentlicher Erfolgsfaktor für Demokratisierung sein. Darüber hinaus sind Freiheitsrechte zentral für Rechtsstaatlichkeit. Der Rechtsstaat unterwirft sämtliches Handeln von Bürgern und dem Staat niedergeschriebenem Recht, welches wiederum der Prämisse universeller Menschenrechte, zu denen fundamentale Freiheitsrechte gehören, unterworfen ist. ${ }^{9}$ Wenn sich Myanmar also tatsächlich in Richtung Demokratie bewegt hat, dann muss man spätestens seit 2011 auch eine positive Entwicklung von praktizierten Freiheitsrechten erkennen können.

Mindestens formal gab es diese Entwicklung. Die Militärjunta, das so genannte State Law and Order Restoration Council (SLORC), hatte ursprünglich mit dem Dekret Nummer 1 die Ausübung fundamentaler Freiheitsrechte im Jahr 1988 verboten. Diese Rechte wurden jedoch mit der Verabschiedung der neuen Verfassung 2010 wiedereingeführt. In Autokratien ist es allerdings nicht unüblich, dass Freiheitsrechte auf dem Papier, zum Beispiel in der Verfassung, gewährt werden, aber versucht wird, deren praktische Ausübung zu verhindern und $\mathrm{zu}$ bestrafen. ${ }^{10}$ Deshalb ist es wichtig, einen Blick speziell auf die Umsetzung von Freiheitsrechten $\mathrm{zu}$ werfen, welche insbesondere durch Protestversammlungen sichtbar wird. Protestversammlungen setzen sowohl Meinungsund Versammlungsfreiheit, als auch Vereinigungsfreiheit voraus, da sich Menschen, um protestieren zu können, organisieren, versammeln und ihre Meinung artikulieren müssen. Zudem lassen sie sich von Außenstehenden „beobachten“ (damit also auch „messen“). Proteste sind daher gut als Indikator für die praktische Ausübung von Freiheitsrechten geeignet.

5 Dahl 1971.

6 Merkel 2004.

7 Habermas 1989.

8 Unter anderem sind 25\% der Parlamentssitze Militärangehörigen vorbehalten (und stehen nicht zur Wahl). Außerdem unterstehen die Ministerien für „Home Affairs, Border“, und „Defence“ dem Militär.

9 Siehe Artikel 19 der Allgemeinen Erklärung der Menschenrechte.

10 Ginsburg/Simpser 2014. 


\section{Wie hat sich Protestaktivität seit 2011 verändert?}

Die folgende Analyse von Protestereignissen greift auf Daten von 185 Protestversammlungen, die zwischen 1. Februar 2011 und 31. Dezember 2014 in Myanmar stattgefunden haben, zurück. Diese wurden über eine Protestereignisanalyse der Nachrichtenwebsite The Irrawaddy gesammelt und durch weitere Quellen quergeprüft. ${ }^{11}$ Mit hoher Wahrscheinlichkeit ist dies jedoch eine nichtrepräsentative Stichprobe, da weder randomisiert werden konnte, noch es sich um eine vollständige Grundgesamtheit von Protesten handelt. Diese Einschränkung betrifft jedoch die meisten Protestereignisanalysen sowie viele nichtexperimentelle Erhebungsverfahren. ${ }^{12}$ Die wissenschaftliche „Best Practice“ ist allerdings, die Datenquellen, potentielle Fehler, sowie das Erhebungsverfahren so transparent wie möglich $\mathrm{zu}$ besprechen, was bei der Dokumentation des Datensatzes nach bestem Wissen und Gewissen befolgt wurde. ${ }^{13}$ Trotz der methodischen Schwierigkeiten lässt sich durch die Triangulation dieser mit anderen Daten und Methoden potentiell eine hohe Validität von Forschungsprojekten erreichen. Deshalb leistet der Datensatz trotz seiner potentiellen Schwächen einen relevanten Beitrag zur Forschung. Das Myanmar Protest Event Dataset ${ }^{14}$ wurde bereits von der GESIS veröffentlicht. Zwar wäre es ideal hier schon Daten bis 2017 mit einbeziehen zu können, allerdings ist die Kodierung von Nachrichtenartikeln entlang über hundert Variablen, die vom eigentlichen Ereignis bis ins Nachhinein (z.B. eventuell späteren Gerichtsurteilen) reichen, ein langwieriger Prozess, der weitere Monate erfordern wird. Da allerdings schon die ersten Jahre nach der Öffnung Myanmars interessante Ergebnisse liefern, sollen diese bereits hier in einer Research Note zur Verfügung gestellt werden.

Tabelle 1 zeigt die Verteilung einiger Hauptvariablen aus dem Datensatz. Es wird bereits auf dem ersten Blick deutlich, dass sich Protestaktivität über die Zeit erhöht hat. Die Anzahl von Demonstrationen und Streiks hat sich von $2011 \mathrm{zu}$ 2014 mehr als vervierfacht. Ebenso hat sich die Größe der Proteste im selben

11 Diese sind bisher die private Myanmar Times sowie der Regierungszeitung The Global New Light of Myanmar. Falls gemeldet wurde, dass Protestteilnehmer im Nachgang verhaftet oder rechtlich belangt werden sollen / wurden, wurden diese Informationen mit Informationen von der Assistance Association for Political Prisoners Burma (AAPPB) überprüft. Genauere Informationen zum „cross-check“ lassen sich der Dokumentation des Datensatzes entnehmen (s. Fußnote 12). 12 Für eine ausführliche Diskussion der Stärken und Schwächen von Protestereignisanalysen, siehe Hutter 2014.

13 Informationen zur Kodierung und Fehlerquellen können dem Methodenreport des Datensatzes, herunterladbar bei der GESIS, entnommen werden.

14 Buschmann 2016, 2017b. 
Tabelle 1: Hauptvariablen (Demonstrationen und Streiks).

\begin{tabular}{|c|c|c|c|c|}
\hline & 2011 & 2012 & 2013 & 2014 \\
\hline$N=185$ & 17 & 62 & 33 & 73 \\
\hline Durchschnittliche Teilnehmerzahl & 530 & 672 & 440 & 1062 \\
\hline Dauer der Proteste (Modus) & $<24 \mathrm{~h}$ & $24-48 \mathrm{~h}$ & 5-7 Tage & 1-2 Wochen \\
\hline $\begin{array}{l}\text { Anzahl Gruppen, genannt als } \\
\text { Organisatoren }\end{array}$ & 3 & 6 & 6 & 30 \\
\hline Protestthemen (gruppiert) & 7 & 12 & 12 & 20 \\
\hline Protestanzahl mit sensiblen ${ }^{1}$ Themen & 1 & 5 & 5 & 22 \\
\hline Serienproteste & $1(5.88)^{2}$ & $25(40.32)^{2}$ & $18(54.54)^{2}$ & $24(32.87)^{2}$ \\
\hline Verhaftungen & 4 & 115 & 51 & 140 \\
\hline
\end{tabular}

1: Beinhaltet: call for justice; forced army recruitment; call for human rights; disapproval with officials; opposition to government plans.

2: Absolute Zahl (relative Zahl).

Zeitraum circa verdoppelt. Von eher kurzfristigen Protesten (unter 24 Stunden) in 2011, waren die meisten Proteste in 2014 länger währende Protestcamps. Das spiegelt sich auch in der Häufigkeit von Serienprotesten wider, also Protesten die im direkten Zusammenhang mit vorherigen Protesten stehen. Was die geografische Verteilung von Protesten angeht zeigt sich, dass zwar - wenig überraschend - die meisten Proteste in Städten (Yangon und Mandalay) stattfanden, allerdings auch in eher ruralen Gebieten protestiert wurde (siehe Abbildung 1).

Diese Kennzahlen haben sich tatsächlich in einer Weise verändert, wie man sie erwarten könnte, wenn fundamentale Freiheitsrechte im Zuge einer Demokratisierung zunehmend gewährt würden. Zudem haben sich nicht nur quantitative Aspekte von Protestaktivität verändert, sondern auch qualitative Charakteristika. Besonders erwähnenswert ist, dass über die Zeit mehr Nichtregierungsorganisationen, soziale Bewegungen und andere Gruppen offen als Organisatoren von Protesten genannt wurden, was zuvor nicht der Fall war. Die NLD, die seit März 2016 in der Regierungsverantwortung ist, sowie ihre YouthOrganisation wurden über alle Jahre hinweg in nur etwa 10\% aller Proteste erwähnt, was deutlich macht, dass es nicht nur die NLD ist, die in Myanmar protestiert. In den Jahren 2013 und 2014 sind außerdem vermehrt s.g. „Single Claim Movements“ als Organisatoren aufgetaucht. Dabei handelt es sich um Gruppen, die nur ein spezielles Ziel verfolgen und sich nur zur Erreichung dieses Ziels gegründet haben. ${ }^{15}$ Das mehr Gruppen genannt wurden, muss natürlich

15 Ein Beispiel hierfür ist das "Committee to Deter Moving of the Gems Marketplace", welches in 2014 die Verlagerung eines Marktes in Mandalay verhindern wollte. 


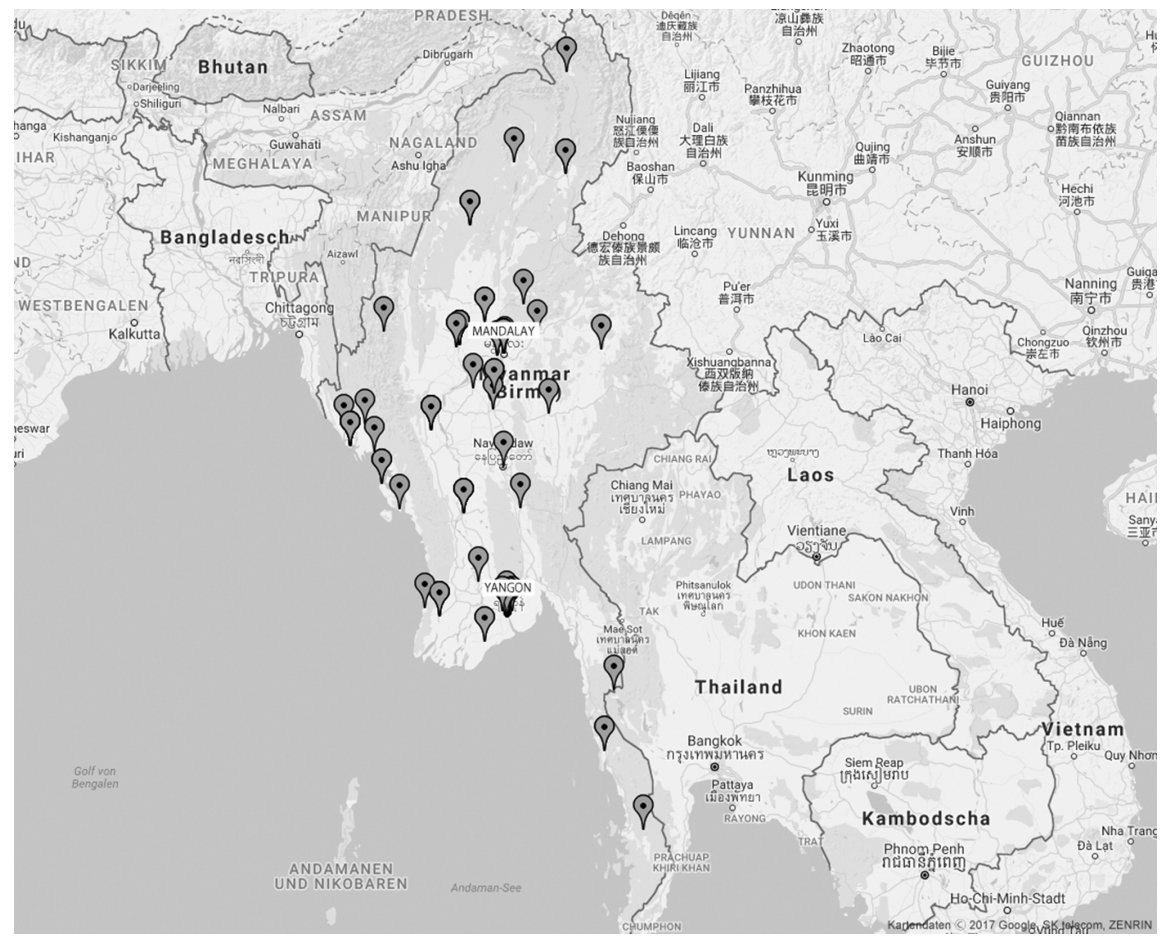

Abbildung 1: Unterschrift: „Geografische Verteilung der Protestereignisse“.

nicht heißen, dass es insgesamt mehr ziviles Engagement gibt, sondern kann auch bedeuten, dass bereits bestehende Akteure „selbstbewusster“ geworden sind und sich offener präsentieren. Beides spräche aber praktisch für mehr Freiraum für zivilgesellschaftliches Engagement.

Thematisch scheint es eine Veränderung hin zu pluralistischeren und kritischeren Themen gegeben zu haben. Speziell seit 2013 wurde zunehmend für Themen wie Menschenrechte und gegen Landraub protestiert. Zusammengefasst lässt sich also erkennen, dass sich der Freiraum für zivilgesellschaftliches Engagement über die Zeit vermutlich tatsächlich vergrößert hat. Es stellt sich jedoch die Frage ob und wie sich das Verhalten des Staates, welches zuvor von Repression gekennzeichnet war, gegenüber Protestteilnehmern gewandelt hat. Zwar impliziert mehr und verändertes zivilgesellschaftliches Engagement bereits, dass Repression wahrscheinlich zurückgegangen ist, Repression kann sich aber auch einfach transformiert haben. 


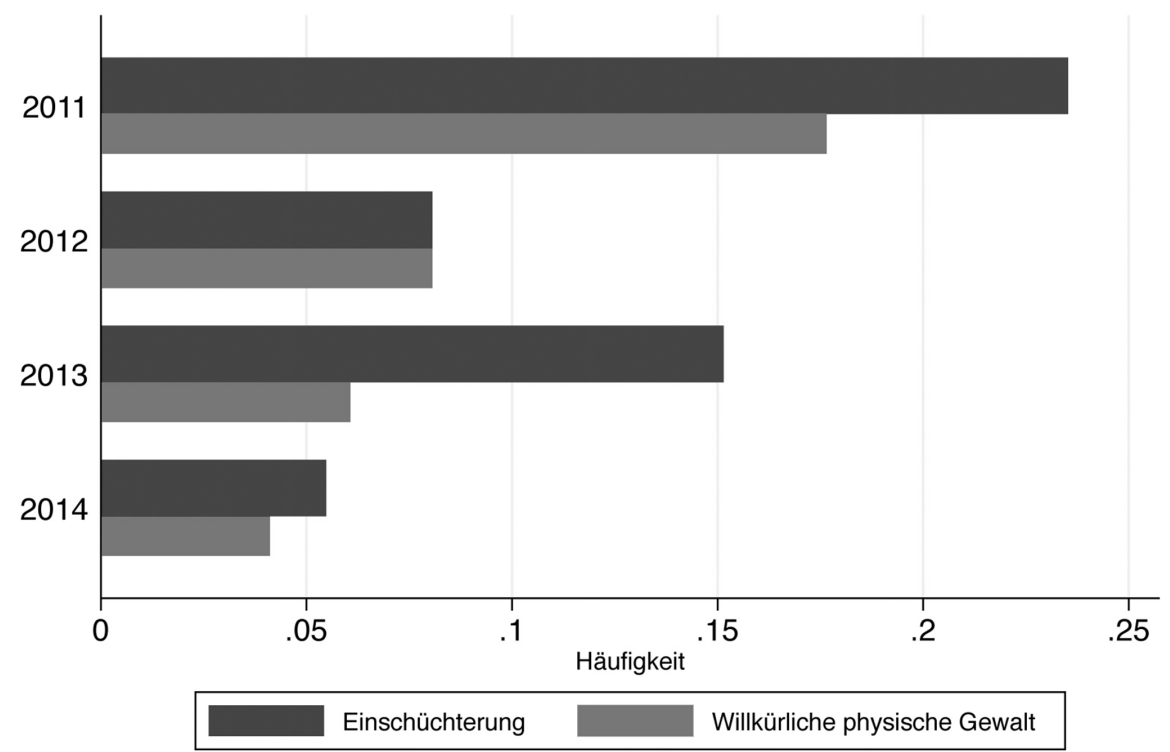

Abbildung 2: Unterschrift: „Informell-autokratische Methoden gegen Protestteilnehmer“.

Wie Abbildung 2 deutlich macht, haben physische Gewalt und die Einschüchterung von Protestteilnehmern mit der Zeit deutlich nachgelassen.

Allerdings waren solche Methoden auch 2014 noch in vielen Fällen vorhanden. Insbesondere die Einschüchterung von Teilnehmern durch Vertreter von staatlichen Sicherheitskräften war zumeist gegenwärtig. Der Ausschlag von „Einschüchterung“ in 2013 betraf vor allem Proteste, die gegen die gewaltsame Auflösung von Protestcamps gegen die Letpadaung Kupfermine im November 2012 protestiert haben. Bis 2014 hat sich die Anwendung dieser informell-autokratischen Methoden jedoch deutlich verringert. Diese Befunde sind zunächst einmal positiv zu bewerten. Stellt man der oberen Grafik allerdings die Anzahl von Protesten mit anschließenden Verhaftungen gegenüber (siehe Abbildung 3), wird ein fast gegenläufiger Trend deutlich:

Verhaftungen auf Grundlage von Gesetzesvergehen sind zunächst einmal ein formal-rechtsstaatliches Mittel. Bei genauerer Analyse der Gesetze, aufgrund derer Protestteilnehmer inhaftiert wurden, zeigt sich jedoch, dass diese weit von internationalen Menschenrechtsstandards entfernt sind (siehe z.B. Artikel 12 des Pyidaungsu Hluttaw Law No. 15/2011, auch bekannt als „Peaceful Assembly and Peaceful Procession Law“). So war es unter anderem bis vor kurzem möglich, 

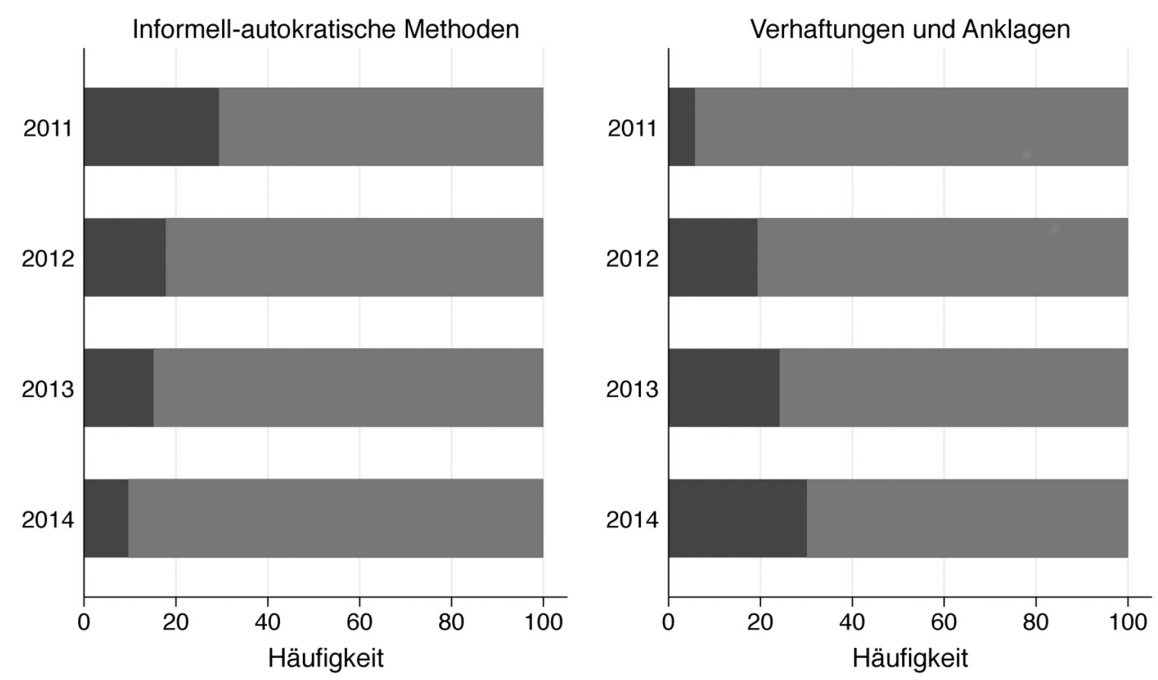

$\mathrm{Ja}$

Nein

Abbildung 3: Unterschrift: „Repression oder Rechtsstaatlichkeit?“

nicht gewalttätige Protestteilnehmer allein für die Verbreitung von „Falschinformationen" mehrere Jahre zu inhaftieren. Obwohl beispielsweise das Peaceful Assembly and Peaceful Procession Law mehrmals reformiert und einige repressive Passagen entfernt wurden, ist die Anzahl der Verhaftungen trotzdem gestiegen. Am 31. Mai 2016 hat das Parlament (Amyotha Hluttaw) die erneute Reform des Peaceful Assembly and Peaceful Procession Laws verabschiedet. Hier bleibt abzuwarten, ob Verhaftungen in Zukunft im Nachgang zu gewaltlosen Protesten abnehmen werden oder lediglich andere Gesetze als Grundlage von Verhaftungen und Verurteilungen genutzt werden. Jüngste Statistiken zu politischen Gefangenen zeigen allerdings, dass zum Beispiel der Paragraph 505b des Strafgesetzbuches oder Paragraph 66d des Telecommunications Laws, die es ermöglichen, Dissidenten mit bis zu drei Jahren Gefängnis zu bestrafen, seit 2016 vermehrt angewandt wurden. ${ }^{16}$ Auch stellt sich die Frage, inwiefern die strafrechtliche Verfolgung von Protestteilnehmern durch Gerichte Ausdruck von Inkompetenz bzw. fehlendem Wissen über Konzepte wie freie Meinungsäußerung und „Rule of Law“ oder tatsächlich intendierter Repression ist. ${ }^{17}$

16 Buschmann 2017a.

17 Siehe hierzu zum Beispiel Cheesman 2015. 


\section{Fortschritt bis Ende 2014... und dann?}

Protestaktivität hat mit der Wahl von Thein Sein zum Präsidenten und den im Anschluss eingeleiteten Reformen sowohl quantitativ als auch qualitativ zugenommen. Zumindest willkürliche Gewalt gegen Protestteilnehmer hat bis Ende 2014 abgenommen, was insgesamt dafür spricht, dass die praktische Ausübung fundamentaler Freiheitsrechte in Myanmar eine positive Entwicklung genommen hat. Dies ist eine notwendige Bedingung für weitere Demokratisierung. Allerdings entsteht auch der Eindruck, dass Protestteilnehmer zunehmend mit Hilfe geltender Gesetze im Anschluss an friedliche Proteste verfolgt werden. Gerade seit den international gefeierten Wahlen in 2015 und dem Amtsantritt der neuen NLD-Regierung in 2016 würde man erwarten, dass auch diese Form der Repression abgenommen hat. Ob dies der Fall ist und wie Protestdynamiken sich ab 2015 verändert haben, wird die Analyse der folgenden Jahre zeigen.

\section{Referenzen}

Buschmann, Andy (2016): Myanmar Protest Event Dataset. Version: 1. GESIS Data Archive. Dataset. http://doi.org/10.7802/1168

Buschmann, Andy (2017a): Myanmar in 2017: Democratic Progress or Authoritarian Rollback? IAPS Dialogue. https://iapsdialogue.org/2017/10/03/myanmar-in-2017-democratic-pro gress-or-authoritarian-rollback/(11/12/2017)

Buschmann, Andy (2017b): Myanmar Protest Event Dataset. Version: 1.1. GESIS Data Archive. Dataset. http://doi.org/10.7802/1442

Chan, Debby Sze Wan (2017): “Asymmetric bargaining between Myanmar and China in the Myitsone Dam controversy: social opposition akin to David's stone against Goliath”. The Pacific Review 30.5: 674-691.

Chang, Paul Y. (2015): Protest Dialectics: State Repression and South Korea's Democracy Movement, 1970-1979. Palo Alto: Stanford University Press.

Chang, Paul Y., Vitale, Alex S. (2013): "Repressive Coverage in an Authoritarian Context: Threat, Weakness and Legitimacy in South Korea's Democracy Movement”. Mobilization: An International Quarterly 18.1: 19-39.

Cheesman, Nick (2015): Opposing the Rule of Law: How Myanmar's Courts Make Law and Order. Cambridge: Cambridge University Press.

Dahl, Robert A. (1971): Polyarchy: Participation and Opposition. New Haven: Yale University Press.

Ekiert, Grzegorz, Kubik, Jan (2001): Rebellious Civil Society: Popular Protest and Democratic Consolidation in Poland, 1989-1993. Ann Arbor: University of Michigan Press.

Egreteau, Renaud (2012): “Burma/Myanmar”. Political Insight 3.2: 30-33.

Egreteau, Renaud (2016): Caretaking Democratization: The Military and Political Change in Myanmar. Oxford: Oxford University Press. 
Ginsburg, Tom, Simpser, Alberto (2014): Constitutions in Authoritarian Regimes. Cambridge: Cambridge University Press.

Habermas, Jürgen (1989): The Structural Transformation of the Public Sphere: An Inquiry into a Category of Bourgeois Society. Cambridge: Polity Press.

Hutter, Swen (2014): “Protest Event Analysis and Its Offspring”. In: Methodological Practices in Social Movement Research. Hrsg. von Donatella Della Porta. Oxford: Oxford University Press, 335-367.

Kim, Sunhyuk (2009): “Civic Engagement and Democracy in South Korea”. South Korean Observer 40.1: 1-26.

Merkel, Wolfgang (2004): “Embedded and Defective Democracies". Democratization 11.5: 33-58. Oberschall, Anthony (2000): "Social Movements and the Transition to Democracy". Democratization 7.3: 25-45. 